\title{
Kernos
}

Revue internationale et pluridisciplinaire de religion grecque antique

23 | 2010

Varia

\section{Theoi sumbômoi et autels multiples}

Réflexions sur les structures sacrificielles partagées

loanna Patera

\section{(2) OpenEdition}

1 Journals

\section{Édition électronique}

URL : http://journals.openedition.org/kernos/1582

DOI : 10.4000/kernos. 1582

ISSN : 2034-7871

Éditeur

Centre international d'étude de la religion grecque antique

\section{Édition imprimée}

Date de publication : 1 janvier 2010

Pagination : 223-238

ISSN : 0776-3824

\section{Référence électronique}

Ioanna Patera, "Theoi sumbômoi et autels multiples», Kernos [En ligne], 23 | 2010, mis en ligne le 10

octobre 2013, consulté le 19 avril 2019. URL : http://journals.openedition.org/kernos/1582 ; DOI :

$10.4000 /$ kernos. 1582 


\title{
Theoi sumbômoi et autels multiples. Réflexions sur les structures sacrificielles partagées
}

\begin{abstract}
Résumé : Des grands autels que se partagent divinités et puissances héroïques aux autels doubles, les structures associées au sacrifice sont généralement analysées en fonction de leur forme. Les autels doubles sont principalement associés à des divinités honorées dans un même espace mais sur des structures différentes, ou encore à la double nature du rite, considérée par certains comme olympienne et chthonienne. En examinant de manière plus approfondie les regroupements de puissances mentionnés par les sources écrites et en les confrontant aux données archéologiques disponibles, nous tenterons de nuancer les associations généralement établies entre les différents types de structures sacrificielles et la nature des rites qui y sont accomplis.
\end{abstract}

Abstract: Sacrificial structures, particularly altars shared by divine and heroic figures and the so-called double altars, are usually analysed on the basis of their form. The double altars are generally associated with divinities honoured in a single space though on separate structures, or with the double nature of the rites, considered by some scholars as being Olympian or chthonian. Through a thorough examination of the groups of divinities mentioned in the written sources and their comparison with the available archaeological data, we will attempt to qualify the established associations between the various types of sacrificial structures and the nature of the rituals that are performed there.

L'étude des associations des dieux grecs a mené à les étudier à l'intérieur même d'un système polythéiste, sur la base notamment des épiclèses des divinités et des fonctions qui les réunissent. Un sujet moins exploré est l'association de dieux qui sont honorés ensemble, dans un même espace ou plus particulièrement sur un même autel. L'exemple des dieux sumbômoi, des dieux qui partagent un autel, mène à l'examen des divinités regroupées autour d'un autel ou d'un rite, et des divinités réparties sur des autels plus nombreux. Le corpus qui suit, loin d'être exhaustif, rassemble des sources épigraphiques et archéologiques, de lieux et d'époques variés, qui permettent d'enrichir les attestations de ces partages. Les structures portent parfois les traces des rites accomplis sur ce type de support. Sans avoir l'ambition de résoudre toutes les questions que posent ces associations divines et la plasticité du polythéisme qu'elles illustrent, cette étude esquissera quelques variations dans le partage de l'espace et des structures rituelles. 
En effet, loin de se cantonner dans des domaines imperméables, les dieux grecs établissent entre eux des partages qui peuvent prendre des formes variées. Ainsi les espaces consacrés abritent parfois de nombreux cultes, bien qu'ils soient généralement attribués à une divinité spécifique. Les dieux peuvent être

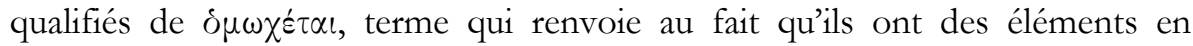

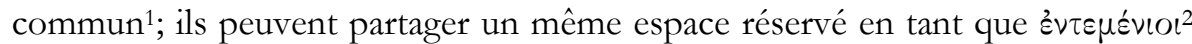

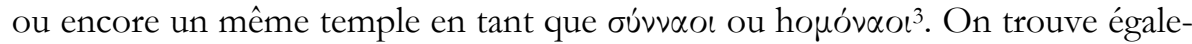

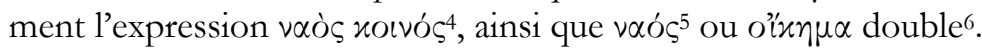

Ce voisinage spatial ne signifie pas pour autant des rites communs. En revanche, les autels destinés à honorer des puissances différentes indiquent des rites susceptibles d'être de nature commune. Que les rites soient ou non communs, la multiplication des autels regroupés dans un même espace permet par ailleurs d'interroger les manières dont se regroupent les dieux. Lorsque les rites qui les honorent sont connus, on peut encore tenter de voir si les autels sont destinés à des pratiques rituelles propres à ces structures. Le cas des autels « doubles », une étroite association de deux autels généralement de forme différente, connus par les données archéologiques mais non attestés par les sources écrites, est censé illustrer des rites de nature différente. D'autres structures enfin, des autels divisés en deux ou plusieurs parties, constituent parfois une allusion à une séparation rituelle spécifique et difficile à cerner.

Les autels ne sont que rarement attribués à une divinité précise par une inscription, bien que nous connaissions parfois en détail les festivités qui encadrent la construction et la consécration d'une structure ${ }^{7}$, son traitement ${ }^{8}$, ce qui y est sacrifié ou déposé9. Certaines attributions nominatives sont connues par les

\footnotetext{
1 Thucydide, IV, 97, 4; en l'occurrence, ils partagent le même sanctuaire.

${ }^{2}$ LSAM 52, A 1.7 (Milet, I ${ }^{e r}$ s. de n.è., vente de la prêtrise d'Asclépios). Sauf indication contraire, les dates s'entendent avant notre ère.

${ }^{3}$ LSCG 60, 1.19 et 20 (Épidaure, ca 400, règlement relatif aux cultes d'Asclépios et d'Apollon; $I G I^{2}$ 1, 41, 1. 2-3).

4 Pausanias, VIII, 32, 3 : temple d'Héraclès et d'Hermès à Mégalopolis.

${ }^{5}$ Paus., VIII, 9, 1 : vaóc double à Mantinée, partagé entre Asclépios d'un côté, Létô et ses enfants de l'autre.

6 Paus., II, 10, 2: dans la première pièce de l'o'́xn $\mu \alpha$ double situé dans le sanctuaire d'Asclépios à Sicyone, il y a une statue d'Hypnos, alors que la pièce du fond, dans laquelle seuls les prêtres peuvent pénétrer, est dédiée à Apollon Karneios.

${ }^{7}$ La paix entre Antioche et Antioche de Kydnos (Tarse), conclue vers 160, est suivie par la construction d'un autel d'Homonoia dans le sanctuaire d'Athéna, pris en charge par les prytanes, cf. LSAM 81, 1. 5-6. Des sacrifices sur cet autel ainsi qu'une procession se déroulent le jour de la consécration, cf. 1. 6-11. Pour une nouvelle fondation d'autel, $c f . L S C G$ 154, B 1. 11-15 (Cos, 1 1e moitié du III $\mathrm{s}$.).

${ }^{8}$ Le plus fameux est le traitement de l'autel de Cyrène, comprenant un nettoyage et une purification après un sacrifice non convenable: LSS 115, A 1. 26-31 (fin du IVe s.). Pour l'entretien des autels, $c f$. E. LuPu, Greek Sacred Law. A Collection of New Documents, Leiden/Boston, 2005 (RGRW, 152), p. 374.

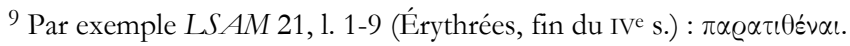


règlements sacrificiels ${ }^{10}$ ou par des dédicaces ${ }^{11}$. D’autres sont spécifiées lorsqu'il s'agit d'accomplir des actions qui requièrent un autel particulier, comme par

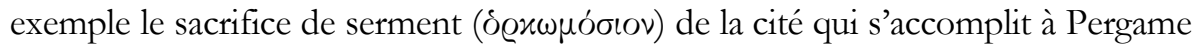
sur l'autel de Zeus Sôtêr situé sur l'agora' ${ }^{12}$.

\section{Autels partagés}

Comme il en va de l'espace de culte, un autel peut être partagé entre plu-

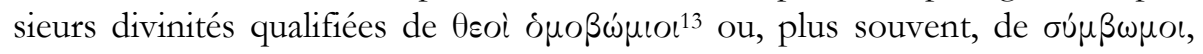
c'est-à-dire des dieux honorés sur un autel commun ${ }^{14}$. Les dieux qui partagent à

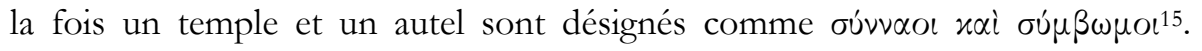

10 Ainsi l'autel d'Apollon Puthios à Athènes : LSCG 25, A (III--II s., Asclépieion, IG II²,

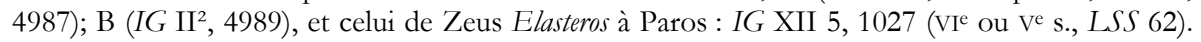
L'inscription de Paros serait d'ailleurs plus récente que l'autel, cf. A. OHNEsorg, Ionische Altäre. Formen und Varianten einer Architekturgattung aus Insel- und Ostionien, Berlin, 2005 (Archäologische Forschungen, 21), p. 30-31. Sur un groupe d'autels de l'Asclépieion d'Athènes, sont spécifiés les gâteaux à sacrifier à Héraclès, cf. LSCG 24 (III $-\mathrm{II}^{\mathrm{e}}$ s., $\left.I G \mathrm{II}^{2}, 4986\right)$; des cippes provenant du Pirée permettent de connaitre les sacrifices pour les Moirai, cf. LSCG 22 (IVe s., IG II'2, 4971); Artémis

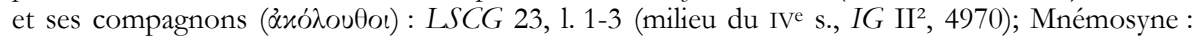
LSCG 26 (Athènes, IVe s.; B.J. MERITT, "Greek Inscriptions », Hesperia 32 [1963], p. 1-56, 46, $\mathrm{n}^{\circ}$ 62). Cf. également LSCG 27 (Athènes, Asclépieion, III ${ }^{\mathrm{e}}-\mathrm{II}^{\mathrm{e}}$ s., IG $\mathrm{II}^{2} 4988$ ).

11 Par exemple, ID 2305 (en partie seulement LSS 55), datée du II s. et provenant de Délos, est une dédicace faite par Damôn d'Ascalon à Zeus Ourios, Astarté Palestinê et Aphrodite Ourania, trois divinités qualifiées d'epêkooi. Elle est gravée sur un autel et précise qu'il n'est pas permis (oủ

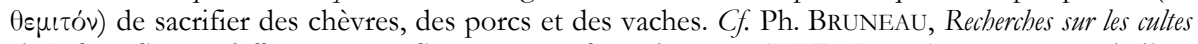
de Délos à l'époque hellénistique et à l'époque impériale, Paris, 1970 (BEFAR, 217), p. 347. Au Phalère, une dédicace gravée sur un autel mentionne Hestia, le Céphise, Apollon Puthios, Létô, Artémis Lochia, les Ilithyies, Achélôos, Kallirhoé, les nymphes Géraistiades genethliai, ainsi que Rhapsô qui est inconnue par ailleurs : cf. LSS 17, 1. 1-6.

12 Ce sacrifice garantit à Asclépiadès et à ses descendants, acquéreurs de la prêtrise d'Asclépios, que tout sera fait conformément aux décisions de la cité : cf. $L S A M 13,1$. 28-29 (avant 133).

13 Thuc., III, 59, 2.

14 Plutarque, De l'amour fraternel, 21 (Mor., 492c) (Héraclès et Iolaos); Propos de table V, 5, 2

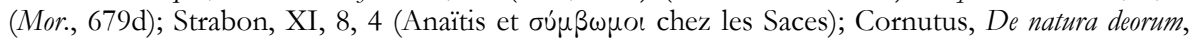

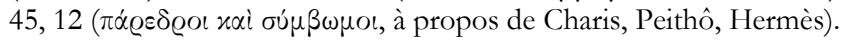

15 Certaines mentions découlent de comparaisons de type général, par exemple du fait de se retrouver en compagnie agréable lors d'un dîner tout comme les dieux qui ont un même autel et

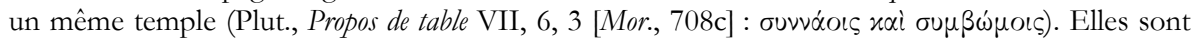
particulièrement fréquentes pour les divinités égyptiennes: IG XI 4, 1223, 1.10-12 (Délos),

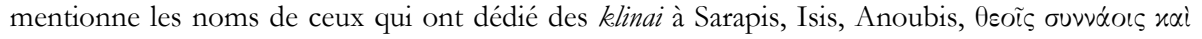

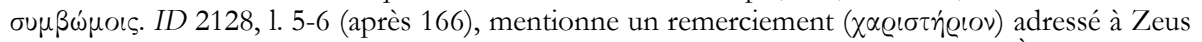

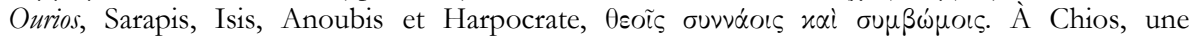

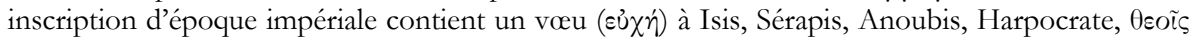

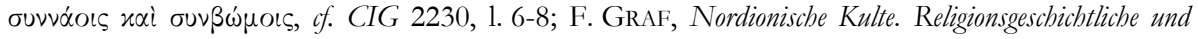
epigraphische Untersuchungen zu den Kulten von Chios, Erythrai, Klazomenai und Phokaia, Rome, 1985, n 65. La même formule apparaît à Délos : ID 2146 (127/6); à Minoa d'Amorgos au II s. : IG XII 7, 255; à Thessalonique : IG X 2, 1, 85 et aussi à Rome: IG XIV, 1007. Des formules similaires apparaissent également à Cos, of. W.R. PATON, E.L. Hicks, The Inscriptions of Cos, Oxford, 1891, n 64; à Éretrie au I ${ }^{\text {er }}$ s. : IG XII Suppl., 565, 1. 11. À Délos (ID 2387, 1. 2-3, après 
Toutefois, le terme oú $\beta \omega \mu$ o n'est pas très fréquent et, jusqu'à présent, n'apparaît dans la documentation épigraphique qu'à partir d'environ 40016. Dès lors, les nombreux autels qui sont communs à deux ou à plusieurs divinités invitent à rechercher des textes faisant référence au partage de façon plus ou moins explicite à défaut d'un terme spécifique. Les règlements stipulent avec une précision variable les dieux honorés sur un autel, comme par exemple celui d'Aphrodite Peithô et d'Hermès à Mytilène daté du II ${ }^{\mathrm{e}} \mathrm{s} .{ }^{17}$. À Épidaure, où l'on trouve un autel d'Apollon et un d'Asclépios, on sacrifie sur le premier à Létô et à Artémis, et sur le second aux hopóvøo du dieu ${ }^{18}$. À côté des sacrifices prescrits à des divinités précises, on rencontre également la formule «à ceux qui sont avec

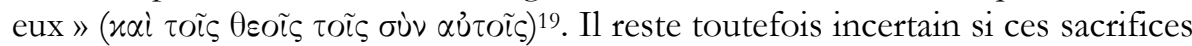

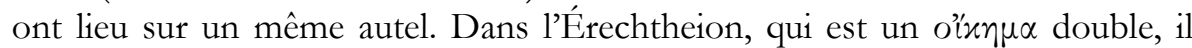
existe, outre les autels individuels de Boutès et d'Héphaistos, celui de Poséidon sur lequel les Athéniens sacrifient aussi à Érechthée ${ }^{20}$. Ailleurs, on trouve dans

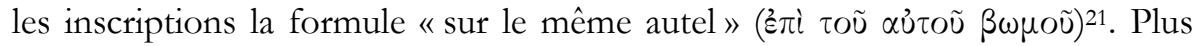

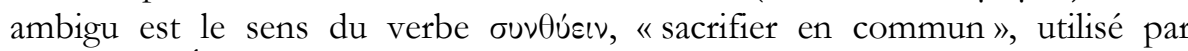
exemple à Érythrées, où la mention du sacrifice d'un £̊ $\mu \eta \tau \eta \dot{s}$ (un gâteau) à Asclépios et à Apollon ne permet pas de savoir si cela se passe sur un seul autel, chacun des dieux possédant le $\operatorname{sien}^{22}$.

L'oracle rendu à Mnésiépès de Paros au milieu du $\mathrm{III}^{\mathrm{e}} \mathrm{s}$. permet de suivre la procédure d'une institution sacrificielle sur un autel nouvellement construit. Après avoir fait construire un sanctuaire d'Archiloque, Mnésiépès doit fonder un

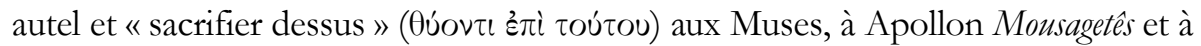
Mnémosyné23. La suite prescrit des sacrifices et des sacrifices de bon augure ( $\theta u ́ \varepsilon \iota \nu$

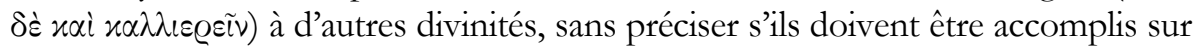

166), un remerciement est adressé à Asclépios, Hygie, Apollon, Létô, Artémis Agrotera et tous les

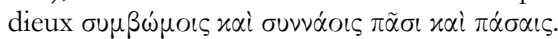

${ }^{16}$ LSS 17, A 1.3 (Phalère, ca 400, IG $\mathrm{II}^{2}$ 4548).

17 LSCG 126, 1. 2-4. V. Pirenne-Delforge, «Le culte de la persuasion. Peithô en Grèce ancienne ", RHR 208 (1991), p. 395-413, 412, n. 73, relève l'ambiguïté qu'il existe dans ce cas entre Aphrodite et Peithô d'une part et Aphrodite Peithô d'autre part.

${ }^{18}$ LSCG 60, 1. 1-6 et 18-23.

${ }^{19}$ LS AM 36, 1. 1-2 et 11-12 (Priène, règlement relatif au culte de Sarapis, ca 200).

${ }^{20}$ Paus., I, 26, 5. LSCG 31, 1. 2-3 (Athènes, Acropole, IVe s., IG II ${ }^{2}$ 1146) prescrit un sacrifice à Poséidon et à Érechthée, sans que l'autel soit précisé. Pour ce double culte, $c f$. S. DARTHOU, «Retour à la terre : fin de la geste d'Érechthée », Kernos 18 (2005), p. 69-83, 79-81.

${ }^{21}$ LSCG 18, A 1. 44-48 (calendrier d'Erchia, $1^{\text {re }}$ moitié du IV s.), concerne le sacrifice à Sémélé. La même expression apparaît en E, 1. 44-46. Selon S. Dow, "The Greater Demarkhia of Erkhia », BCH 89 (1965), p. 180-213, 193, cette mention suivrait celle du sacrifice destiné à Dionysos.

${ }^{22}$ LSAM 24, A 1. 12-13 (380-360) et 1. 34-35 pour l'autel d'Apollon. Pour les sacrifices doubles autour d'Asclépios, $c f$. A. Petropoulou, «Prothysis and altar: a case study », in R. ÉTIENNE, M.-T. Le DinAheT (éds), L'espace sacrificiel dans les civilisations méditerranéennes de l'antiquité. Actes du Colloque tenu à la Maison de l'Orient, Lyon, 4-7 juin 1988, Paris, 1991, p. 25-31, 27.

${ }^{23}$ LSCG 180, 1. 2-4. 
ce même autel ${ }^{24}$. Ensuite, l'oracle prescrit par la même formule de fonder un autel et de «sacrifier dessus » à Dionysos, aux Nymphes et aux Horai. Enfin, il reprend la prescription de sacrifier et de faire des sacrifices de bon augure à d'autres dieux $^{25}$. Il en découle que Mnésiépès doit fonder deux autels ${ }^{26}$.

Les redistributions suivant lesquelles on sacrifie à une divinité sur l'autel d'une autre ou à plusieurs divinités sur un même autel semblent souvent commandées par l'attribution des épiclèses. Il peut s'agir de différentes épiclèses attribuées à une même divinité ou du partage d'une même épiclèse entre plusieurs dieux ${ }^{27}$. À Athènes, le prêtre d'Apollon Pythien se charge du sacrifice accompli dans les Jardins, probablement sur l'autel de Puthios et comprenant une truie adulte pour Apollon Alexikakos, un bovin pour Patrôios, et probablement un bovin pour Apollon Puthios, réunissant ainsi trois figures importantes d'Apollon ${ }^{28}$. Outre la répartition sur les autels selon les épiclèses des divinités honorées, on peut supposer que les liens de parenté jouent également un rôle important, comme par exemple à Magnésie du Méandre où le douze du mois Artemisiôn, lors de la fête instituée en l'honneur de Zeus Sôsipolis après la conclusion du traité de paix avec Milet, on sacrifie à Zeus Sôsipolis, à Artémis Leukophruênê et à Apollon Puthios: le plus beau bélier est offert à Zeus, une chèvre à Artémis et un bouc à Apollon. Cette association dans la fête se modifie au niveau du sacrifice. Le sacrifice à Zeus a lieu sur l'autel de Zeus Sôsipolis, ceux à Artémis et Apollon sur celui d'Artémis ${ }^{29}$. Lors d'une fête en l'honneur de la divinité qui assure la sauvegarde de la cité on honore aussi sa patronne ${ }^{30}$. Ainsi les sacrifices sont répartis sur les autels suivant d'une part les liens de parenté et d'autre part suivant la place qu'occupent ces divinités dans le cadre particulier de la cité.

${ }^{24}$ LSCG 180, 1. 4-6, à Zeus Huperdexios, à Athéna Huperdexia, à Poséidon Asphaleios, à Héraclès et à Artémis Eukleia.

${ }^{25}$ LSCG 180, 1. 9-12 : à Apollon Prostatêrios, à Poséidon Asphaleios, à Héraclès.

${ }^{26}$ LSCG 180, 1. 17-19.

${ }^{27}$ De façon générale, on peut renvoyer pour ce sujet à l'analyse de P. BRULÉ, « Le langage des épiclèses dans le polythéisme hellénique (l'exemple de quelques divinités féminines) ", Kernos 11 (1998), p. 13-34. Ce critère de redistribution s'observe dans l'attribution de nombreux autels déliens, par exemple ceux de Zeus Polieus et Athéna Polias, Zeus Sôtêr et Athéna Sôteira. Pour un inventaire des sacrifices sur les autels déliens, of. R. ÉTIENNE, «Espaces sacrificiels et autels déliens », in ÉTIENNE, LE DiNAHET (éds), o.c. (n. 22), p. 75-84, 80-84.

${ }^{28}$ LSS 14, 1. 52-55 (règlement relatif au culte d'Apollon, 129/8, SEG 21, 469). Ces sacrifices ont très probablement lieu pendant les Thargélies. Cf. R. PARKER, Athenian Religion. A History, Oxford, 1996, p. 95-96 pour Apollon Pythien, et p. 64 pour Apollon Patrôios.

${ }^{29}$ LSAM 32, 1. 48-53 (après 185/4).

30 Artémis Leukophruênê est en effet l'archêgetis de la cité, cf. LSAM 33, A 1. 18. 


\section{Autels associés à des groupes de dieux}

Outre les autels de tous les dieux comme ceux de Lykosoura ${ }^{31}$ et d'Olympie ${ }^{32}$, on trouve des exemples d'autels destinés à des groupes particuliers. Sur le grand autel de l'Amphiaraeion d'Oropos, on sacrifie à Amphiaraos et à ceux dont le nom est inscrit sur la structure même ${ }^{33}$. Selon Pausanias, l'autel est divisé en cinq parties dont chacune est assignée à des puissances spécifiques : la première est réservée à Héraclès, Zeus et Apollon Paiôn; la deuxième est allouée à des héros et à leurs femmes; la troisième appartient à Hestia, Hermès, Amphiaraos et son fils Amphilochos; la quatrième est assignée à Aphrodite et à Panakeia, à Iasô, à Hygie et à Athéna Paiônia, et la cinquième aux Nymphes, à Pan ainsi qu'aux fleuves Achélôos et Céphise ${ }^{34}$. Cette description est étayée par deux stèles du IV $\mathrm{s}$. dont l'une mentionne Amphiaraos, Amphilochos et peut-être aussi Hermès, l'autre Hestia $^{35}$. Les rapprochements entre les différentes puissances ont reçu des traitements significatifs, leurs rapports ont été discutés et des parallèles ont été trouvés $^{36}$. Ce qui importe ici, c'est le réseau cultuel de ce regroupement dans lequel il n'y a pas de séparation entre dieux et héros ${ }^{37}$, et où la nature des puissances et celle du rite qui leur est adressé ne constitue pas un facteur de distinction.

Un dispositif similaire s'observe dans l'Asclépieion de $\operatorname{Cos}^{38}$, où les plaques de revêtement de l'autel le plus ancien du site portent les noms de quatre puissances : Halios, Hamera, Machaon et Hécate. S. Sherwin-White y a vu un ensemble « hétéroclite » et une sélection privée de puissances ${ }^{39}$, malgré les liens

31 Selon Paus., VIII, 37, 10, au-delà de l'alsos consacré à Despoina, il y a des autels de Poséidon Hippios et d'autres dieux. Une inscription précise que le dernier autel est celui de tous les dieux.

32 Paus., V, 15, 10.

33 Paus., I, 34, 5. Il semble d'ailleurs que l'on sacrifie l'animal de son choix, cf. LSCG 69, 1. 30-31.

${ }^{34}$ Paus., I, 34, 3.

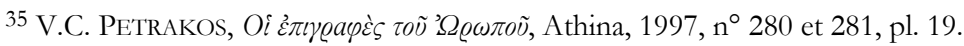

${ }^{36}$ La première triade constituée par Héraclès, Zeus et Apollon Paiôn, a été mise en parallèle avec un oracle cité par Démosthène, Contre Midias (21), 52, prescrivant des sacrifices et des prières à Zeus Hupatos, à Héraclès et à Apollon Prostatêrios: of. A. VerbanCK-PIÉRARD, "Les héros guérisseurs: des dieux comme les autres! À propos des cultes médicaux dans l'Attique classique ", in V. Pirenne-Delforge, E. SuÁrez De la Torre (éds), Héros et héroines dans les mythes et les cultes grecs, Liège, 2000 (Kernos, suppl. 10), p. 281-332, 321. Toutefois, les épithètes des divinités de ces triades ne sont pas identiques; l'importance des épiclèses dans l'attribution des sacrifices est évidente en Magnésie du Méandre : of. LSAM 32, 1. 48-53. Voir encore P. SINEuX, Amphiaraos. Guerrier, devin et guérisseur, Paris, 2007, p. 86-89 et 142-147.

37 VerbanCK-PiéraRd, l.c. (n. 36), p. 323.

38 R. Herzog, Kos. Ergebnisse der deutschen Ausgrabungen und Forschungen, Berlin, 1932, p. 56, date cette inscription du milieu du IVe s. Selon S.M. SHERwIN-White, Ancient Cos. An Historical Study from the Dorian Settlement to the Imperial Period, Göttingen, 1978 (Hypomnemata, 51), p. 334, n. 395, elle remonte plutôt au dernier quart du IV $\mathrm{s}$. Les orthostates ont été réutilisés dans l'autel hellénistique d'Asclépios. Cf. SEG 41, 688.

39 S.M. SHERWIN-WHITE, o.c. (n. 38), p. 335. 
qu'entretiennent entre elles ces figures ${ }^{40}$. Cependant, la comparaison avec l'autel d'Oropos suggère que ce genre de regroupement pouvait aussi être officiel et donc public.

\section{Regroupements d'autels}

Si plusieurs divinités peuvent partager un même autel, certains sanctuaires foisonnent en structures sacrificielles. Il faut dès lors tenter de cerner les logiques suivant lesquelles elles sont regroupées.

Les Asclépieia semblent particulièrement bien fournis en autels. À Épidaure, le prêtre fait le tour de tous les autels ${ }^{41}$. Une inscription mentionne séparément celui d'Apollon sur lequel le prêtre brûle probablement des parfums ${ }^{42}$, puis celui d'Asclépios, et enfin des petits autels, des $\beta \omega \mu$ íro $\iota^{43}$. Au Pirée, les inscriptions gravées sur les quatre faces d'un bloc donnent des informations sur les différents sacrifices préliminaires de gâteaux qui doivent être accomplis. La face A porte une liste des divinités qui reçoivent des popana: Malétas, Apollon, Hermès, Iasô, Akesô et Panakeia, les chiens et les meneurs de chiens ${ }^{44}$. On lit également qu'Euthydémos d'Éleusis, prêtre d'Asclépios, a consacré les stèles qui se trouvent près des autels et sur lesquelles il a le premier représenté les popana requis ${ }^{45}$. Les faces $\mathrm{B}$ et $\mathrm{C}$ prescrivent les sacrifices pour Hélios et Mnémosyné et indiquent l'existence de trois autels sur lesquels on ne verse pas de libation de vin ${ }^{46}$. Il se peut que ces trois autels soient ceux près desquels Euthydémos a consacré des stèles, mentionnés par la face A. Comme le remarque à juste titre S. Scullion, chaque destinataire semble ici être honoré sur son autel par des

40 Voir de façon générale J.W. RIeThmüLler, Asklepios. Heiligtümer und Kulte I, Heidelberg, 2005, p. 216 et n. 789. Halios et Hamera apparaissent encore côte à côte sur un autel inscrit daté du III s. : cf. M. SEGRE, Iscriz̧ioni di Cos, Roma, 1993, p. 176, EV 18b 1. Hélios reçoit des sacrifices à l'Aslépieion du Pirée (IG $\mathrm{II}^{2}, 4962$, cf. n. 46). Halios et Asclépios apparaissent ensemble sur un autel circulaire inscrit de Tégée, daté du I ${ }^{\mathrm{er}} \mathrm{s}$. de n.è. (IG V 2, 82). Machaon, considéré comme le fils d'Asclépios (notamment dans l'Iliade, IV, 193-194), apparaît dans les listes des dieux invoqués dans les cultes médicaux : cf. Hérondas, Mimes IV, 9. Un xoanon d'Hécate se trouve dans la stoa de l'Asclépieion de Titané, à côté de ceux de Dionysos, Aphrodite, la Mère des dieux et Tyché (Paus., II, 11, 8). Sur les liens entre Hécate et Hélios, en particulier dans les sources littéraires, $c f$. R. VON RudLOFF, Hekate in Ancient Greek Religion, Winnipeg, 1999, p. 78-80.

${ }^{41} \operatorname{LSS} 25, \mathrm{~A} 1.3$ (Épidaure, $\mathrm{II}^{\mathrm{e}}-\mathrm{III}^{\mathrm{e}}$ s. de n.è., IG IV 2 1, 742). Cette même action apparaît chez Aristophane, Ploutos, 679 .

42 LSS 25, A 1. 14.

43 LSS 25, D 1. 35. Une inscription de Lampsaque, datée du $\mathrm{II}^{\mathrm{e}}$ s., mentionne des autels au pluriel situés dans le temenos, tout en précisant que le prêtre doit faire brûler de l'encens sur l'autel d'Asclépios, of. $L S A M$ 8, 1. 19-20 et 29. À Cos, au IVe s., le sacrifice aux Nymphes se fait "sur les autels », cf. LSCG 152, 1. 1-4.

${ }^{44}$ LSCG 21, A 1. 3-10 (IG II ${ }^{2}$ 4962). Chacun reçoit trois popana. Cette partie de l'inscription qui est la plus récente, date du début du $\mathrm{IV}^{\mathrm{e}} \mathrm{s}$.

45 LSCG 21, A 1. 14-17.

46 LSCG 21, B 1. 18-28; C 1. 29-31. 
offrandes qui lui sont propres ${ }^{47}$. Toutefois, la plupart du temps les inscriptions mentionnent des autels au pluriel, sans préciser à qui ils appartiennent ${ }^{48}$.

Dans l'importante concentration d'autels que l'on trouve à Olympie, les soixante-neuf ou soixante-dix exemples mentionnés par Pausanias appartiennent à un nombre variable de divinités ${ }^{49}$. Certaines en possèdent plusieurs; on peut citer notamment Zeus, Artémis, Apollon, Athéna, Héra mais aussi $\operatorname{Pan}^{50}$. L'appartenance commune d'un autel peut être attribuée à la communauté d'une épiclèse : ainsi Zeus Laoitas en partage un avec Poséidon Laoitas. Toutefois, ce critère ne s'applique pas de façon systématique et les diverses divinités qui portent l'épiclèse Hippios ou Hippia, à savoir Poséidon, Héra, Arès et Athéna, n'ont pas d'autel commun. D'autres dieux partagent un autel tout en étant les propriétaires exclusifs d'un autre ${ }^{51}$. Il ne s'agit pas de regroupements familiaux à proprement parler; par exemple Héraclès Parastatês et son frère Idas ont chacun leur autel, et il en va peut-être de même pour les autres frères: Epimédès, Paiônios et Iasos. Parfois, le partage révèle le recours à un générique regroupant les dieux afin de s'adresser à une totalité, ou bien de n'en oublier aucun: on attribue par

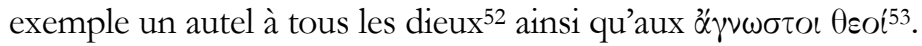

Bien qu'un mécanisme de regroupement de divinités sur la base de la fonction ou des liens familiaux puisse apparaître dans certains cas, chaque logique entrevue appelle immédiatement son contre-exemple. La cohérence qui apparaît dans un type de sources est démentie, ou reformulée, lorsque des données différentes sont prises en compte. Que penser du fait que Déméter et Corè, mère et fille souvent honorées ensemble, ne partagent pas nécessairement leur autel ? À Éleusis, elles ont deux autels distincts ${ }^{54}$. À Lykosoura, Despoina ne partage son autel ni avec Déméter ni avec la Grande Mère, divinités que l'on a trop souvent tendance à identifier entre elles ${ }^{55}$.

\footnotetext{
47 S. SCulLiON, «Olympian and Chthonian », ClAnt 13 (1994), p. 75-119, 81. Notons cependant que le nombre des autels ne correspond pas à celui des divinités évoquées.

48 Ainsi par exemple au Pythion d'Athènes: LSS 14, 1.33 (129/8).

${ }^{49}$ Paus., V, 14, 4.

${ }^{50}$ De la même façon, Hélios en possède plusieurs sur l'Acrocorinthe : Paus., II, 4, 6.

51 Ainsi Alpheios partage un autel avec Artémis, Dionysos avec les Charites et Hermès avec Apollon.

52 Paus., V, 14, 8; V, 15, 1.

53 Paus., V, 14, 8. Des autels pour les dieux inconnus sont aussi mentionnés au Phalère (I, 1, 4).

54 K. Clinton, Eleusis. The Inscriptions on Stone. Documents of the Sanctuary of the Two Goddesses and Public Documents of the Deme, Athens, 2005, $\mathrm{n}^{\circ} 177,1.203$ (329/8, IG II' 2 1672); concernant l'emplacement de ces autels, of. id., "Sacrifice at the Eleusinian Mysteries ", in R. HägG, N. Marinatos, G.C. NordQuist (éd.), Early Greek Cult Practice. Proceedings of the Fifth International Symposium at the Swedish Institute at Athens, 26-29 June 1986, Stockholm, 1988, p. 69-79, 71-72.

55 Paus., VIII, 37, 2-3. Ces trois autels ont été identifiés lors des fouilles archéologiques, $c f$. M. Jost, Sanctuaires et cultes d'Arcadie, Paris, 1985, p. 174 pour les références.
} 


\section{Regroupements de structures sacrificielles et rites}

Qu'en est-il du rite dans ces partages? Le support des sacrifices est rarement défini dans les règlements religieux. Celui de l'Asclépieion de Pergame, daté du $\mathrm{II}^{\mathrm{e}}$ s. de n.è., donne en revanche des instructions aux patients qui souhaitent passer la nuit dans le sanctuaire, avant la consultation. La plupart du temps, le lieu et la structure du rite ne sont pas précisés pour les sacrifices signalés par l'inscription. Celui d'un porcelet de lait pour Asclépios est à accomplir sur l'autel. Le soir, trois gâteaux sont à déposer, deux sur la thumelê externe, structure sacrificielle distincte de l'autel d'Asclépios, pour Tyché et Mnémosyné et le troisième pour Thémis dans l'egkoimêterrion ${ }^{56}$, le lieu où se couchent les patients pour voir en rêve le diagnostic du dieu et la cure à suivre. L'autel lui-même est réservé au sacrifice animal, alors que les gâteaux sont répartis sur d'autres structures. Dans ce cas, les diverses structures sacrificielles sont destinées à des divinités et à des matières sacrificielles différentes.

Les cas où plusieurs supports sont utilisés lors d'un même sacrifice sont plus rares. Selon le calendrier de Cos, composé après le synécisme de 366, la veille du sacrifice du bœuf à Zeus Polieus, on brûle un porc et ses viscères sur l'autel en faisant sur eux une libation de lait et de miel, puis on brûle à côté les intestins lavés ${ }^{57}$. Ce sacrifice où l'on brûle tout forme une séquence distincte, souvent considérée comme éloignée du "sacrifice olympien réglementaire ${ }^{58}$. Qu’il s'agisse de marquer le caractère «normal» ou «exceptionnel» du rite ${ }^{59}$ ou d'accomplir un rite conforme à la nature de la divinitée ${ }^{60}$, il est important de souligner que le sacrifice requiert deux lieux distincts, l'autel et l'espace à son côté.

Le sacrifice du porc est suivi de celui du bœuf accompli le lendemain. Le lieu n'est pas précisé mais il s'agit sans doute de l'autel. De la farine, des pains et «ce

\footnotetext{
56 M. WÖRRLE, « Die lex sacra von der Hallenstrasse (Inv. 1965, 20)», in C. HABICHT (éd.), Die Inschriften des Asklepieions, Berlin, 1969 (Altertümer von Pergamon, VIII, 3), $\mathrm{n}^{\circ}$ 161, p. 167-190, 1. 6-11. Cf. F. SOKOLOwSKI, «On the New Pergamene Lex Sacra», GRBS 14 (1973), p. 407-413.

${ }^{57}$ LSCG 151, A 1. 32-34 (milieu du IVe s.). Notons que d'après S. SCULLION, « Sacrificial Norms, Greek and Semitic: Holocausts and Hides in a Sacred Law of Aixone », in P. BRULÉ (éd.), La norme en matière religieuse en Grèce ancienne, Liège, 2009 (Kernos, suppl. 21), p. 153-169, 158, n. 15, les intestins sont simplement lavés à côté de l'autel. La séparation ne doit pas nécessairement être comprise comme un jugement de valeur d'après lequel les intestins seraient indignes d'être déposés sur l'autel, comme le suggère B. LE GUEN-POLLET, «Espace sacrificiel et corps des bêtes immolées. Remarques sur le vocabulaire désignant la part du prêtre dans la Grèce antique, de l'époque classique à l'époque impériale », in ÉTIENNE, Le DiNAHET (éds), o.c. (n. 22), p. 13-23, 14.

58 Une «powerful action » selon la terminologie de M.H. JAMESON, « Notes on the Sacrificial Calendar from Erchia », BCH 89 (1965), p. 154-172, 164-165, qui compare ce sacrifice à celui de Zeus Meilichios rapporté par le calendrier d'Erchia.

${ }^{59}$ F. Graf, «Milch, Honig und Wein. Zum Verständnis der Libation im griechischen Ritual », in Perennitas, studi in onore di Angelo Brelich, Roma, 1980, p. 209-221, 217-218.

${ }^{60}$ SCULLION, l.c. (n. 47), p. 82.
} 
qui doit être enrobé » sont alors sacrifiés sur l'bestia, structure distincte de l'autel ${ }^{61}$. Dans le quatrième fragment du calendrier de Cos, qui se réfère aux activités d'un mois dont le nom n'est pas préservé, une chèvre est sacrifiée aux Charites et reçoit un traitement particulier qui implique un autel et une pierre située au milieu des oliviers ${ }^{62}$. La prêtresse ${ }^{63}$ arrose trois fois l'autel, probablement de sang puis, par un quatrième mouvement la dite pierre; on sacrifie deux portions composées de gâteaux, de viandes et de viscères, en les posant sur l'autel. La suite implique Asia qui serait honorée en tant que mère des Charites et dont le culte est inconnu par ailleurs. Si ses honneurs étaient concentrés sur la pierre comme on l'a supposé64, la chèvre serait destinée à la fois aux Charites et à Asia, donc à des puissances honorées probablement sur des structures distinctes. Ses viscères sont effectivement déposés à la fois sur l'autel et sur la pierre.

Plusieurs structures sacrificielles sont donc parfois utilisées lors d'un même sacrifice, soit que les matières sacrificielles sont destinées à des puissances différentes, soit que des parties de l'animal sacrifié sont traitées différemment sur des structures indépendantes. La multiplication des dispositifs est également attestée par des données archéologiques qui ont généralement été attribuées à la double nature du rite, olympienne ou chthonienne, bien que nous n'ayons pas, dans ce cas, la possibilité d'identifier les mêmes variations rituelles qui apparaissent dans les inscriptions.

\section{Vestiges d'autels « doubles »}

Lorsque des autels se trouvent côte à côte, on a tendance à les attribuer à des divinités distinctes ou bien à leur assigner une fonction différente dans le rite, en particulier s'ils ne présentent pas la même forme ${ }^{65}$. Certains autels sont

61 LSCG 151, A 1. 47-48. Le partage du bœuf est analysé par J. SVENBRO, « Le partage sacrificiel selon une loi sacrée de Cos (IVe s. avant J.-C.) ", in La découpe et le partage du corps à travers le temps et l'espace, 1987 (Anthropozoologica, n spéc. 1), p. 71-76. Aussi, lors du sacrifice en l'honneur de Héra Argeia Heleia Basileia, ce qui doit être enrobé dans la peau de la génisse choisie est brûlé avec un gâteau sur le foyer du temple. Les parts peuvent être emportées, cf. LSCG 151, B 1. 5-10. Sur ce passage, of. G. EKROTH, The Sacrificial Rituals of Greek Hero-Cults in the Archaic to the Early

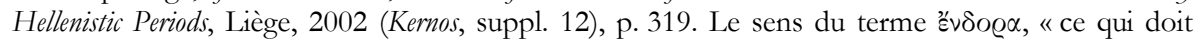
être enrobé dans la peau », est ambigu et mériterait un examen approfondi.

62 LSCG 151, D 1. 5-15.

63 Il ne s'agit pas d'un prêtre, comme le traduisent P.J. RHODES, R. OsBORNE, Greek historical Inscriptions, 404-323 B.C., Oxford, 2003, p. 305 ( $\mathrm{n}^{\circ}$ 62), mais d'une prêtresse.

64 V. Pirenne-Delforge, «Les Charites à Athènes et dans l'île de Cos », Kernos 9 (1996), p. 195-214, 210-212. Ce sacrifice est assimilé à celui du serment des éphèbes. Cf. également RHODES, OSBORNE, o.c. (n. 63), p. 311.

65 Selon J. RuDHARDT, Opera inedita. Essai sur la religion grecque \& Recherches sur les Hymnes orphiques, P. Borgeaud, V. Pirenne-Delforge (éd.), Liège, 2008 (Kernos, suppl. 19), p. 121, lorsqu'un sanctuaire inclut de nombreux autels, ceux-ci sont «adaptés aux différents sacrifices que des communautés d’importance inégale ou que des particuliers viennent y célébrer ». 
d'ailleurs considérés par les modernes comme formant des paires, des « autels doubles », bien que ces derniers ne soient pas attestés dans les sources écrites. Concernant ces associations de structures, on considère encore très souvent que l'animal serait égorgé et brûlé sur l'autel selon un rite dit olympien alors que le sang serait versé dans un bothros, selon un rite souvent considéré comme chthonien et/ou héroïque ${ }^{66}$.

Cependant, les dieux peuvent être honorés sur le même autel que les héros et les rites qui leurs sont adressés sont souvent identiques $^{67}$. La distinction entre nature chthonienne et olympienne du rite qui a longtemps guidé les historiens de la religion grecque ne semble opératoire $^{68}$, ni pour ce qui est de la nature de la puissance honorée ni pour ce qui est de la forme des struc-

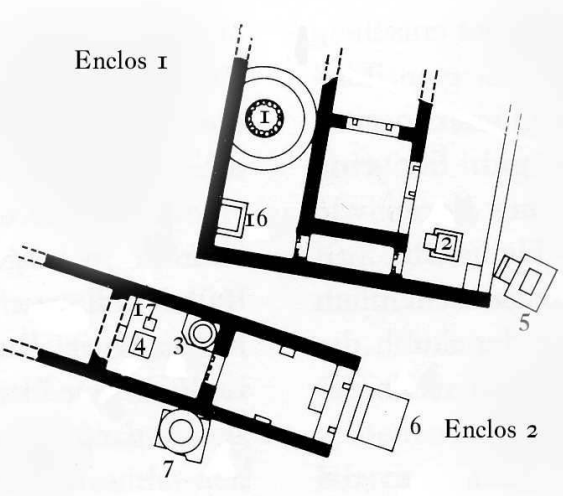

Fig. 1 tures.

Les exemples les plus significatifs d'autels « doubles » se rencontrent dans le sanctuaire d'Agrigente, connu dans les travaux archéologiques comme le «sanctuaire chthonien » (Fig. 1)69. La plupart des autels qui nous intéressent sont répartis dans deux enclos qui sont divisés en trois parties par des murs. Ils sont considérés comme constituant des paires, composées d'un autel massif et d'un autel creux ${ }^{70}$. Cependant, dans le cas où l'un des deux autels est situé à l'exté-

\footnotetext{
66 F. ROBERT, Thymélè. Recherches sur la signification et la destination des monuments circulaires dans l'architecture religieuse de la Grèce, Paris, 1939 (BEFAR, 147), p. 167.

67 On peut renvoyer à ce sujet à l'étude de EKROTH, o.c. (n. 61), et notamment à sa conclusion, p. 341.

${ }^{68} \mathrm{La}$ question avait déjà été abordée et nuancée par A. FAIRBANKS, "The Chthonic Gods of Greek Religion », AJPh 21 (1900), p. 241-259. L'historiographie a été traitée par R. SCHLESIER, "Olympische Religion und chthonische Religion», in U. BIANCHI et al. (éds), The Notion of "Religion" in Comparative Research, Rome, 1994, p. 301-310. Leurs conclusions, confirmées par de nombreuses études que nous ne pouvons citer ici, n'ont toutefois pas trouvé l'écho qu'elles méritent.

69 La première utilisation cultuelle du site remonte à la fin du VII ${ }^{\mathrm{e}} \mathrm{s}$. avec la création des enclos qui étaient peut-être à ciel ouvert, cf. D. PANCUCCI, «I Temenoi del santuario delle divinità ctonie ad Agrigento », in M.-J. Fontana, M.-T. Piraino, F.P. Rizzo (éds), Miscellanea di studi classici in onore di Eugenio Manni, 5, Roma, 1980, p. 1665-1676, 1673-1674.

70 V. Hinz, Der Kult von Demeter und Kore auf Sizilien und in der Magna Graecia, Wiesbaden, 1998 (Palilia, 4), p. 82. Des autels doubles se retrouvent à nouveau dans l'aire sacrificielle située au sud des enclos, qui mériterait d'être examinée pour elle-même.
} 
rieur de l'enclos ${ }^{71}$, le dispositif ne permet pas la même interprétation d'un rite conjoint comme pour ceux qui se trouvent côte à côte. À moins d'imaginer un rite comme celui rapporté dans le calendrier de Cos avec d'un côté le sacrifice du bœuf vraisemblablement sur l'autel et de l'autre de la farine, des pains et « ce qui doit être enrobé » brûlés sur l'hestia ${ }^{72}$.

Il est important de noter que ces autels servaient à des rites divers ${ }^{73}$. Parmi les autels creux, certains contenaient des objets mais ne présentaient pas d'autres traces rituelles ${ }^{74}$. Un autel creux de l'enclos 2 est censé avoir servi aux offrandes de nourriture et de libations uniquement en raison de sa position à l'étroit, dans l'angle d'une pièce. L'ouverture (ou botbros) juste à côté contenait de la céramique du IVe $\mathrm{s} .{ }^{75}$. L'interprétation de ces autels creux en tant que lieux d'un «sacrifice chthonien » laisse donc à désirer. L'autel situé au milieu de cette même pièce servait de support à des rites divers, l'un impliquant la combustion et l'autre impliquant le dépôt d'objets, en l'occurrence en bronze ${ }^{76}$.

Des dispositifs constitués de deux autels présentant des formes différentes et portant des traces rituelles variées se rencontrent dans d'autres sanctuaires attribués à Déméter et Corè. Dans la cour du temple du IVe-IIII s. situé à Monte Adranone ${ }^{77}$, il y a un petit autel rectangulaire massif (A2). À l'angle S-O du temple, un autre autel rectangulaire mais creux, est fait de stèles dressées (A3) ${ }^{78}$. Une paire d'autels, cette fois circulaires, se trouve au nord du temple de S. Biagio à Agrigente. L'un est massif et porte des traces de feu (A1) alors que

${ }^{71}$ Comme par exemple l'autel carré 2, associé à l'autel carré creux 5 situé à l'extérieur de l'enclos 1.

${ }^{72}$ LSCG 151, A 1. 47-48.

${ }^{73} \mathrm{Il}$ ne s'agit pas d'un rite chthonien et d'un autre qui serait olympien, comme le suggère la conception commune, soutenue notamment par C.G. YAvIs, Greek Altars. Origins and Typology, Including the Minoan Mycenaean Offering Apparatus. An Archaeological Study in the History of Religions, Saint Louis, 1949, p. 74; PANCUCCI, l.c. (n. 69), p. 1669, n. 24.

${ }^{74} \mathrm{Par}$ exemple l'autel circulaire 1 dans la pièce occidentale de l'enclos 1.

75 Il s'agit de l'autel 3, dans la pièce médiane de l'enclos 2. Cf. HinZ, o.c. (n. 70), p. 82.

${ }^{76}$ L'autel 4 est composé d'un bloc carré avec deux dépressions sur la face supérieure, dont l'une portait des traces de feu et l'autre des ossements carbonisés, des cendres et quelques objets en bronze. Un autre autel double, le 17 dans la même pièce, probablement daté du IVe s., est fait de trois petites pierres cylindriques à la surface creuse, placées sur un bloc de pierre. À côté, une fosse (ou bothros) est délimitée par des dalles de pierre dressées; elle contenait des coupes et des petites lampes. Les traces autour de cette installation suggèrent des sacrifices par le feu : $c f$. P. MARCONI, Agrigento arcaica. Il santuario delle divinità cbtonie e il tempio detto di Vulcano, Roma, 1933, p. 27. Le «bothros» est généralement considéré comme étant de date postérieure, notamment de la fin du IVe s., cf. PANCUCCI, l.c. (n. 69), p. 1672; C. ZOPPI, Gli edifici arcaici del santuario delle divinità ctonie di Agrigento. Problemi di cronologia e di architettura, Torino, 2001 (Mnemè. Documenti, culture, storia del Mediterraneo e dell'Oriente Antico, 2), p. 23-24.

${ }^{77}$ HiNZ, o.c. (n. 70), p. 135-137.

${ }^{78}$ Un parallèle assez proche de cette disposition est attesté à Sabucina, en Sicile centrale, $c f$. ibid., p. 139-141. 
l'autre présente un puits qui contenait des objets (A2) ${ }^{79}$. La variété des formes et des traces rituelles des autels ne peut dès lors se résumer à la dichotomie d'un rite olympien et chthonien. Dans ce cadre de structures sacrificielles dédoublées, on peut d'ailleurs penser à des rites comme le sacrifice préliminaire du porc cité dans le calendrier de $\operatorname{Cos}^{80}$, où l'animal et ses viscères sont brûlés sur l'autel et les intestins à côté.

Les autels doubles n'apparaissent pas uniquement dans ces sanctuaires que l'on a trop rapidement étiquetés comme «chthoniens ». À l'Héraion du Cynthe à Délos, un autel rectangulaire $\mathrm{du} \mathrm{IV}^{\mathrm{e}} \mathrm{s}$. est doublé d'une fosse formant une petite enceinte carrée à son angle $\mathrm{N}-\mathrm{E}^{81}$. Tous deux sont situés à l'extérieur du péribole. Les autels doubles ne présentent d'ailleurs pas nécessairement des formes différentes. Parallèlement aux deux autels d'Oropos recouverts par le grand autel du IV $\mathrm{IV}^{\mathrm{e}}{ }^{82}$, deux autres se trouvent devant l'Héraion à l'embouchure du Sele. Ils datent de la fin du VIe s., sont de forme rectangulaire et de taille légèrement différente ${ }^{83}$. Deux autels monumentaux à prothysis, quadrangulaires et parallèles, mais qui ne sont pas contemporains, ont été retrouvés sur l'agora de Cyrène ${ }^{84}$. Le groupe de cinq autels rectangulaires, alignés et parallèles, qui se trouve sur la terrasse du temple de Déméter à Pergame, se compose d'un grand autel suivi d'une paire symétriquement placée et de deux autres isolés, plus à l'Est $^{85}$. Cet exemple montre à quel point il est réducteur d'associer systémati-

79 Cf. J.A. DE WAELE, Acragas Graeca. Die historische Topographie des griechischen Akragas auf Sizilien, I. Historischer Teil, Groningen, 1971, p. 199-200; HINZ, o.c. (n. 70), p. 74-76.

${ }^{80}$ LSCG 151, A 1. 32-34.

81 A. Plassart, Les sanctuaires et les cultes du mont Cynthe, Paris, 1928 (EAD, 11), p. 206-208.

82 Sur les deux petits autels du dernier quart du ve s., de taille inégale, of. V.C. PETRAKOs, 'O

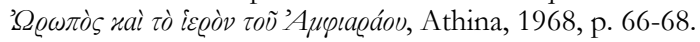

83 P. Zancani Montuoro, U. Zanotti Bianco, Heraion alla foce del Sele. I. Il santuario, il tempio della dea, rilievi figurati varii, Roma, 1951, p. 38. Le plus grand était associé à la trouvaille d'un couteau en fer. Voir aussi G. GreCO, "Strutture per un sacrificio », in G. GreCO, B. FERrARA (éds), Doni agli dei. Il sistema dei doni votivi nei santuari. Atti del Seminario di Studi, Napoli 21 aprile 2006, Napoli, 2008, p. 29-48, 30 et 38. Aucun d'eux n'est situé dans l'axe du temple.

84 S. STUCCHI, « Gli altari marmorei nell'Agorà di Cirene », in ÉTIENNE, LE DiNAHET (éds), o.c. (n. 22), p. $175-178,175$.

85 C.H. BoHTZ, Das Demeter-Heiligtum, Berlin, 1981 (Altertümer von Pergamon, XIII), p. 51-55. Tous les autels datent de la première phase du temple alors que le noyau du grand autel est peut-être plus ancien. À l'époque romaine, vers le $\mathrm{II}^{\mathrm{e}}$ s. de n.è., un grand nombre de petits autels a été ajouté sur la terrasse; ils étaient dédiés aux Thesmophores, à Zeus Ktêsios, à Hermès Diaktoros, au Panthéon, à Hélios; cf. C.M. Thomas, «The Sanctuary of Demeter at Pergamon: Cultic Space for Women and its Eclipse », in H. Koester (éd.), Pergamon. Citadel of the Gods, Archaeological Record, Literary Description, and Religious Development, Harrisburg, 1998 (Harvard Theological Studies, 46), p. 277-298, 293. Pour le grand autel A inscrit d'une dédicace de Philétairos, son frère Eumène et sa mère Boa (283-263), cf. S. KASPER, "Zum großen Altar der Demeterterrasse in Pergamon », in Pergamon, Gesammelte Aufsätze, Berlin, 1972, p. 70-93, en particulier 91 pour la datation. Une structure parfois identifiée comme un bothros (cf. BOHTZ, o.c., p. 52-53) se trouve dans l'angle nord de l'autel A, ensuite recouvert par l'autel de Philétairos. Un autre puits se trouve sur le côté sud du grand autel. 
quement les autels doubles à une dichotomie rituelle alors que le nombre des structures sacrificielles peut démentir ce schéma binaire.

\section{Vestiges d'autels divisés}

Outre ces dédoublements ou multiplications d'autels, les structures sacrificielles clairement divisées en deux pour former un dispositif spécifique sont rarement considérées dans les études qui dressent une typologie des autels. Un foyer entouré de pierres est construit à l'extérieur du

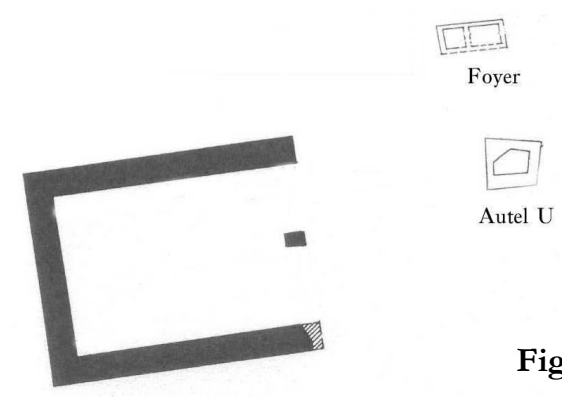

Fig. 2 temple de Kommos en Crète (Fig. 2) ${ }^{86}$. Il présente une surface double constituée de deux enclos carrés dont l'un, à l'Est, était déjà largement détruit lors de sa découverte alors que l'autre, à l'Ouest, contenait encore des cendres ${ }^{87}$. Ce foyer est postérieur à l'autel rectangulaire et creux $(\mathrm{U})$ qui se trouve plus au sud. Celui-ci a été construit vers 700 , lors de la deuxième phase du temple $\mathrm{B}$, et contenait des ossements brûlés ${ }^{88}$. Toutefois, il est resté en fonction même après la construction du foyer. Il est impossible de savoir si la division de ce dernier concernait le nombre des divinités honorées ou le rite. Un autre type de division apparait sur l'autel situé dans l'axe du temple C à l'époque hellénistique (ca 375). Il supportait en effet des ossements mais aussi une figurine de taureau séparée du feu par une dalle qui constituait peut-être une division momentanée ${ }^{89}$.

Plusieurs dispositifs à compartiments se rencontrent aussi dans le sanctuaire d'Apollon à Cyrène. La surface supérieure de l'autel de Panakeia comporte un

\footnotetext{
${ }^{86}$ Notons que tous les autres foyers, situés à l'intérieur, étaient certainement utilisés pour la cuisine et pour brûler des parts du sacrifice : $c f$. J.W. SHAW, M.C. SHAW et al., Kommos IV. The Greek Sanctuary, I, Princeton, New Jersey, 2000, p. 679. Il y avait en effet des ossements brûlés, des restes de poissons (associés en particulier au foyer 4) et des escargots sur tous les foyers du temple C (p. 683-684).

${ }^{87}$ Ibid., p. 676-677.

88 Selon la typologie commune, l'autel U est un bothros ou un autel « hollow ceremonial».

${ }^{89}$ Le nombre des autels a fait penser que chacun d'entre eux était destiné à une ou plusieurs divinités : cf. SHAw, SHAW et al., o.c. (n. 86), p. 716. Bien qu'il y ait de nombreux candidats sur le site (un relief de Pan du $I^{\text {er }}$ s. de n.è. [S 197], une inscription du $\mathrm{II}^{\mathrm{e}}$ s. qui mentionne Zeus et Athéna [ $\left.\mathrm{n}^{\circ} 76\right]$ et un autel circulaire portatif de Poséidon du II $\mathrm{s}$. [n $\mathrm{n}^{\circ} 75$, trouvé dans le temple C]), il n'est pas évident que la construction d'un nouvel autel marque l'introduction d'une nouvelle divinité, comme le suggèrent les fouilleurs : ibid., p. 674.
} 
encastrement pour un double brasier amovible portant des inscriptions ${ }^{90}$. Le brasier d'un autre autel est quadruple et porte les noms des divinités incisés sur le bord antérieur de la surface sacrificielle ${ }^{91}$. La rareté de ces brasiers amovibles a fait penser qu'ils constituaient une particularité cyrénéenne ou qu'ils n'avaient pas été conservés ailleurs. En revanche, la division des surfaces, également rare, n'est pas un élément local comme le montre l'exemple de Kommos.

\section{Conclusion}

La complexité de l'image donnée par ces autels n'a d'égale que la complexité des panthéons eux-mêmes. Divers dieux ont un autel commun et le partagent aussi avec des héros, démentissant ainsi la séparation des rites de nature olympienne et chthonienne. En outre, il est impossible de savoir combien de divinités étaient honorées sur un même autel, si elles recevaient des sacrifices communs ou si les diverses parties réservées des autels recevaient des sacrifices particuliers. Pour donner un exemple des variations dans les associations on peut citer le cas des cultes des Douze dieux ${ }^{92}$, honorés sur un seul autel à l'agora $\mathrm{d}^{\prime}$ Athènes ${ }^{93}$, par deux sur six autels à Olympie ${ }^{94}$ ou par triades sur quatre autels à Délos ${ }^{95}$. Outre les variations dans les regroupements qui peuvent éclairer les theoi sumbômoi, il faut tenir compte de la variation des rites accomplis sur les autels. Ces variations impliquant un animal, des végétaux ou des objets deviennent significatives dans un sanctuaire comme celui d'Agrigente. Ses autels doubles, si tant est qu'ils forment des paires, ne peuvent être interprétés par la simple combinaison d'éléments olympiens et chthoniens.

90 C. PARISI PRESICCE, «Cirene: gli altari del santuario di Apollo », in ÉtIENnE, Le DinAHET (éds), o.c. (n. 22), p. 159-165, 164 et fig. LI b. Cf. aussi fig. LI c.

91 Ibid., fig. LI d. Sont inscrits les noms d'Hécate, Hygie, Panakeia et Héraclès. Parmi ces autels comportant de nombreux encastrements, les plus anciens remontent au début du IVe s. et se situent, comme l'exemple cité ici, dans la partie orientale du sanctuaire d'Apollon, occupée par le temenos d'Aphrodite (p. 164).

92 Par exemple $L S A M$ 2, 1. 9-10 (III' s., règlement d'un thiase de Chalcédoine); LSAM 32, 1. 43-44 (Magnésie du Méandre, 185/4). Cf. S. Georgoudi, «Les Douze Dieux des Grecs : variations sur un thème », in S. GEORGOUDI, J.-P. VERNANT (éd.), Mythes grecs au figuré, de l'antiquité au baroque, Paris, 1996, p. 43-80. La relation des Douze à d'autres puissances divines ou héroïques est examinée par S. GEORGOUDI, «Les Douze Dieux et les autres dans l'espace cultuel grec », Kernos 11 (1998), p. 73-83.

93 H.A. Thompson, R.E. Wycherley, The Agora of Athens, Princeton, New Jersey, 1972 (The Athenian Agora, 14), p. 129-136 et fig. 34; la chronologie est réexaminée par L.M. GADBERY, «The Sanctuary of the Twelve Gods in the Athenian Agora: a revised view », Hesperia 61 (1992), p. 447489, 467-469.

94 Sur ces autels, of. Apollodore, II, 7, 2; Pindare, Ol. 5, 5 et 10, 23. Selon le grammairien

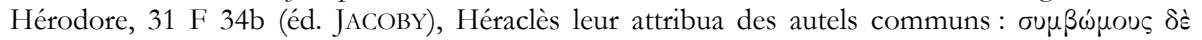

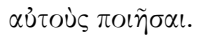

95 Éd. WiLL, Le Dodécathéon, Paris, 1955 (EAD, 22), p. 178-179. La seule inscription qui mentionne une triade concerne Athéna, Zeus, Héra (p. 155). 
Cette exploration préliminaire, qui ne permet pas de dégager une logique systématique dans les regroupements des puissances, a tenté de les considérer en rapport avec des rites accomplis sur des autels variés. La mise en contexte, selon les régions et les époques, à l'intérieur de panthéons locaux, ainsi que les différences qui apparaissent entre les récits mythiques et les sources qui éclairent les pratiques rituelles, pourraient peut-être, dans une enquête plus approfondie, donner un autre sens à ces associations que la seule dichotomie «olympien/ chthonien » qui prévaut encore trop souvent.

Ioanna PATERA

Universität Erfurt

Max Weber Kolleg

Am Hügel 1

DE - 99084 Erfurt

Courriel:ioanna.patera@gmail.com

\section{Liste des figures}

Fig. 1. Les enclos du «sanctuaire chthonien» d'Agrigente. D'après V. HINZ, Der Kult von Demeter und Kore auf Sizilien und in der Magna Graecia, Wiesbaden, 1998 (Palilia, 4), p. 80, fig. 12 .

Fig. 2. Le temple B de Kommos en Crète. D'après J.W. SHAw, M.C. SHAw et al., The Greek Sanctuary, I, Princeton, New Jersey, 2000 (Kommos, IV), pl. I.30. 